\title{
Development of Tactile Display and an Efficient Approach to Enhance Perceptual Analysis in Rehabilitation
}

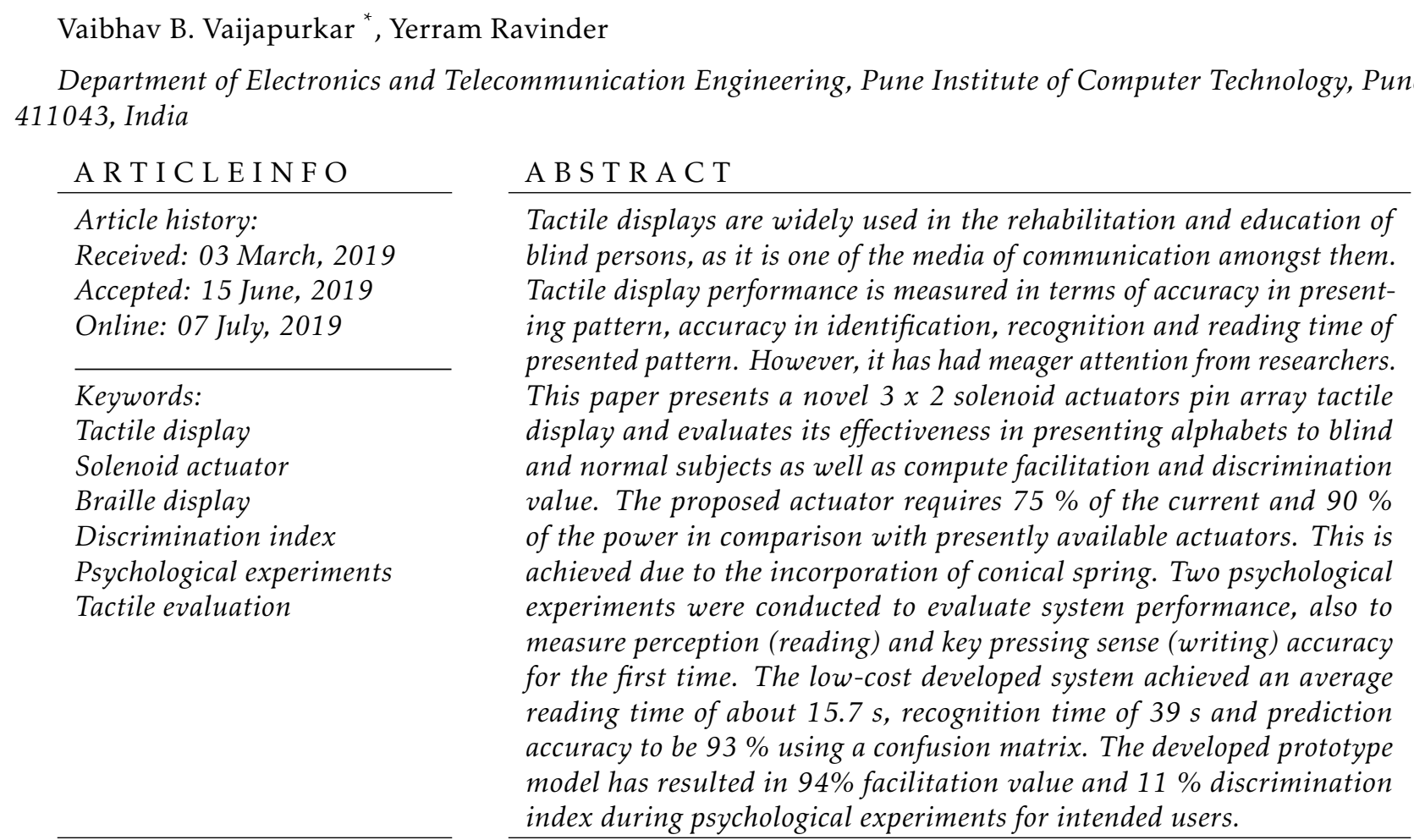

\section{Introduction}

Tactile devices have proved their value in educating blind persons. In the human sensory system, skin plays a significant role in touch and feel. A visually disabled person uses predominantly uses touch and feel as a medium to communicate. The tactile display is developed using a different kind of actuator such as a piezoelectric, solenoid, electromagnetic, Shape memory alloy (SMA) wires and spring, soft gel, etc. The various performance parameters of tactile devices are reading speed, recognition rate, and perception accuracy. Miniaturization of such devices obviously has challenges and limitations in fabricating and assembling. A diverse range of technology is used to develop tactile displays.

A sixty-four elements tactile display using shape memory alloy (SMA) wires and 8 tactel modules was developed reported by [1]. A piezoelectric bimorph was used to form 6 and 8 dot Braille cells. Touching force varying between 0.03 to $0.39 \mathrm{~N}$ was reported by [2]. A portable eBook reader for the blind with 10
Braille cells using a piezoelectric ultrasonic actuator was presented in [3]. Thermal bimorph microactuator was developed by [4] and was analyzed for changing mechanical dimensions rather than changing the input voltage to reduce power consumption. A vibrotactile display using a pen-like haptic interface to represent textures on it was also developed. Different textures were represented and $80.83 \%$ accuracy was reported by [5]. Xiaosong $\mathrm{Wu}$ et al. have devised a portable pneumatically actuated refreshable Braille cell. A 2 X 3 micro-bubble actuator by UV lithography process formed to achieve $0.56 \mathrm{~mm}$ displacement [6].

A compact planar distributed tactile display was developed by Ki-Uk Kyung. Also, observed the effect of frequency on texture judgment through the developed display. The correct answer reported is dependent on the stimulus 90-90\% times. Small-scale shapes, patterns, and gratings have conveyed good tactile information to subjects; the correctness achieved is $90 \%$. Stimuli with higher frequencies have shown the worst discrimination of patterns as reported in [7]. Makoto Takamiya et al. proposed a low power and flexible

\footnotetext{
${ }^{*}$ Corresponding Author: Vaibhav B. Vaijapurkar, Department of Electronics and Telecommunication Engineering, Pune Institute of Computer Technology, Pune, 411043, India, vaibhav.vaijapurkar@gmail.com
} 
Braille sheet with a plastic actuator. It uses back-gated OFETs SRAM technology to enhance speed [8]. The speed of Braille sheet display was improved to 1580 times that of the speed without SRAM. H. R. Choi et al. used the tactile display as a Braille display. The frequency by which the dots activate is 15-60 Hz [9]. Achieved average recognition rate for HRR is $60 \%$ while for NRR, it is $40 \%$. T. Matsunaga et al. fabricated and developed 2-D and 3-D tactile pin display to represent the character and graphics information dynamically using an SMA micro-coil actuator [10]. Christopher R. Wagner et al. used RC servomotors to develop a tactile shape display. The $6 \times 6$ pin array tactile display was developed with $1 \mathrm{~mm}$ pin diameter; The result showed that displacement is limited by the slew rate [11]. Masashi Konyo et al. artificially created a soft gel tactile feel display. The most sensitive frequency was found to be $180 \mathrm{~Hz}$ close to physiology [12]. Also, signals with varying shapes such as sinusoidal, triangular, rectangular and sawtooth waves confirmed that rectangular is the most significant signal and the sinusoidal wave is the next most significant. The stimuli of a compound signal had shown a significant tactile feel.

Pruittikorn Smithmaitrie designed and analyzed a piezoelectric Braille display. The touching force of the reader while sensing a Braille character on the tactile display was measured by [13]. Ramiro Velaquez et al. created a tactile binary information display using an SMA actuator. The designed actuator was excited by a 200-500 mA current to achieve a displacement of $3 \mathrm{~mm}$ [14]. Michael J.Mosley et al. proposed the dynamics of an SMA wire bundle actuator through design [15]. Jun Su Lee and Stepan Lucyszyn have developed a refreshable Braille cell using micromachining. They developed electrothermally controlled actuators forming a $3 \times 2$ Braille cell consuming an average power of $0.30 \mathrm{~W}$ and an actuation time $50 \mathrm{~s}$ [16].T. Nobels et.al. had designed high power density electromagnetic actuators for a portable Braille display. It used small linear electromagnetic actuators mounted on a computer mouse displaying 3 characters one at a time [17]. Sarah F. and Paul B proposed a four-level fingertip search display for the blind using 64 solenoids. Overall power consumption was $30 \mathrm{~W}$. It was difficult to control the displacement of each actuator uniformly with the suggested method [18]. K. Deng and E. T. Enikov used electromagnetic localization and actuation to present an on shape display. A prototype module of $4 \times 4$ was developed to experiment with the perception of curvature shapes. The size of the actuator was analyzed to determine its minimal diameter. It had an outer diameter of $1.5 \mathrm{~mm}$, which is suitable for right perception [19].

Different 84 devices were compared with their functionality and readiness levels by [20]. It was observed that only $19 \%$ of total the developed systems were commercialized and others were available in prototype forms. Also, many of them were not evaluated to test performance parameters of a developed unit. In recent years, a portable device with a tactile keyboard and the refreshable display is patented by [21] consist of a touch-sensitive surface, Braille tactile display, and processing unit. The developed unit uses a touch-sensitive screen used to form a tactile pattern on the Braille display. A single piezoelectric Braille cell display is proposed and patented by [22] uses MOSFET drives to magnetize each piezo-actuator to form an expected pattern.

Granit and Eduardo investigated text recognition using six channel vibrotactile wearable display. They experimented skin sensation for the letter, word, and short sentences. The reported recognition accuracy is $82 \%$ and interaction duration 38 seconds. It is observed that repetition in such experiments improves accuracy reported in [23]. Daniele and Claudio designed refreshable Braille display recently [24]. It includes four Braille cells actuated by a solenoid and external magnetic field. It was operated on the $9 \mathrm{~V}$ battery to achieve $1 \mathrm{~mm}$ displacement. Zhiyun Lu had developed a bistable electroactive polymer one cell tactile display. This display is capable of representing Braille letters using air pressure [25]. The author reported $100 \mathrm{mmHg}$ air pressures to raise dot to achieve $0.7 \mathrm{~mm}$ height. It uses $90 \mathrm{~V}$ supply to raise each dot.

Although great efforts have been put into developing such devices throughout the globe; there are some issues that need to be resolved for it to be converted into a product. The accuracy in reading Braille is reported to be $80 \%$ [5] and $90 \%$ [7], while recognition rate is about $60 \%[9]$. As mentioned in [26]; reading/ perception accuracy, recognition rate, and reading speed are the performance parameters for tactile displays. The above review reveals that although different types of tactile displays have been developed, to the best of our knowledge, meager attention has been paid towards performance parameters. Evaluation of developed tactile displays needs to be given more attention for finding accuracy, recognition rate, reading speed.

A survey was conducted in a blind school to understand the usage of electronic gadgets in teaching. It showed that the computer is one of the most commonly used tools but it has a dependency on the school laboratory hence, it is required to have a handheld device to read and write Braille letters. This display caters to the needs of disabled such as training, rehabilitation, and education through play and entertainment. Few more problems raised by participants were, dots remain unraised after frequent use of Braille on paper, that creates difficulty in reading. It is difficult to carry such a document or book because of its weight and size. The device should be easily portable during their primary education.

This paper presents; a low-cost design and implementation of a novel tactile display and its parametric performance evaluation. The designed display is capable of displaying alphabets and numbers in bilingual; effectively experimented during the evaluation of it. The proposed display is the least power consuming prototype as compared with literature. The tapered spring used to actuate is one of its kind and novel, none of the authors reported such spring for actuation over a 
linear spring. Also, it has achieved maximum displacement in the least time compared with a similar type of actuators mentioned in the literature. The developed unit advances in identification accuracy and reading rate. It addresses the key pressing sense of subjects with very high accuracy; which is tested first time in existing work. To support the results; 25 blind and 25 normal subjects are evaluated to test the effectiveness and confirmed use of the display for education. Facilitation and discrimination analysis of experiment finding is done and it confirms desired values. Solenoid actuators are robust, high speed and fast response actuators as compared to the other actuators. It is a tool for educating and training visually impaired persons in their primary stage with or without the help of a trainer. Such systems are also useful in rehabilitation of motor system disorders in humans [27].

Section I presents a detailed study of the available literature to understand the current scenario in the space of the proposed work. Section II presents the development of a six solenoid actuators tactile display and discusses; improvement in solenoid operation with modified spring design. The discussion covers the organization of six solenoids to make a $3 \times 2$ Braille cell. Section III covers the evaluation of the developed tactile display with psychological experiments aimed at understanding the performance parameter. A statistical tool called confusion matrix technique was used to find percentile accuracy in prediction and mis-prediction. Item analysis was used to assess the discrimination index and facilitation value of the developed technique. Performance of device along with the results of the experiments is discussed in section III.

\section{Design and development of tac- tile display}

The micro solenoid actuator available in the market works on $12 \mathrm{~V}$ and requires $0.5 \mathrm{~A}$ current to actuate to have a displacement of $4 \mathrm{~mm}$. The power consumed by one such actuator is $6 \mathrm{~W}$, which is higher compared to piezoelectric or electromagnetic actuators. Tactile display requires six such actuators to form a Braille cell, consuming the power of $36 \mathrm{~W}$. First challenge in the development was to reduce this power consumption. Following steps were taken to develop a more efficient tactile actuator.

\section{Design of a solenoid actuator}

2. Testing of a single actuator

3. Performance test of the designed actuator

4. Control system design

\subsection{Design of a solenoid actuator}

Solenoid actuator has four major components as a metal rod, compression spring, coil, and housing. New actuator design includes the design of a rod which will move vertically after excitation and represents a Braille dot, a compression spring which will move a rod back to its original position after excitation is removed, a coil which will generate an electromagnetic field to hold the rod at the un-raised position once excited. It was required to reduce current consumed by an actuator and hence compression spring design was important. This spring helps actuator to come back to the normal position when the actuator was not actuated. The stainless steel metal rod that moves vertically has a diameter of $2.9 \mathrm{~mm}$ and length of 4.2 $\mathrm{mm}$. The conical spring was designed using music wire. The use of linear spring $[1,5,9,10,18,24]$ is limiting the performance of the actuator hence, the conical spring instead of linear is proposed to use the first time to improve its performance. The proposed spring provides a constant spring rate and improves displacement; which is verified through experimentation. The wire of a diameter $0.15 \mathrm{~mm}$ available in music wire type was selected to generate a stroke of $4 \mathrm{~mm}$. The free length of the spring was $9 \mathrm{~mm}$ with 7 active coils. The housing was designed using iron material press fit assembly. Two sides are kept open for natural cooling. This spring is capable of generating a deflection force of $1 \mathrm{~N}$ showed in Table 1

Table 1: Proposed conical spring parameters.

\begin{tabular}{|c|c|c|}
\hline Parameter(symbol) & Value & Unit \\
\hline Wire diameter $(\mathrm{d})$ & 0.15 & $\mathrm{~mm}$ \\
\hline Small OD(SOD) & 5.8 & $\mathrm{~mm}$ \\
\hline Large OD(LOD) & 7 & $\mathrm{~mm}$ \\
\hline Number of active coils $\left(N_{a}\right)$ & 7 & - \\
\hline The free length $\left(l_{f}\right)$ & 9 & $\mathrm{~mm}$ \\
\hline Density of material $(\rho)$ & 8000 & $\mathrm{Kg} / \mathrm{m}^{3}$ \\
\hline Spring constant $(\mathrm{k})$ & 94.8 & $\mathrm{~N} / \mathrm{mm}$ \\
\hline Pitch of leads $\left(P_{s}\right)$ & 2 & $\mathrm{~mm}$ \\
\hline Shear stress $(\tau)$ & 2.16 & $\mathrm{MPa}$ \\
\hline Deflection force $(\mathrm{F})$ & 1 & $\mathrm{~N}$ \\
\hline
\end{tabular}

\subsection{Testing of a single actuator}

A single solenoid actuator was evaluated to test performance parameters, such as displacement, the current required for actuating to achieve desired displacement, the time required to actuate, etc. The experiment carried out to test this included a DC power supply which can supply $5 \mathrm{~V}$ and $500 \mathrm{~mA}$ current. The electrical parameters are listed in Table 3, It has shown that total power consumed by one existing actuator is $2.5 \mathrm{~W}$ and the proposed actuator is $0.6 \mathrm{w}$. The power consumption is very low as compared with displays developed by [14, 18, 24] for a similar purpose. However total displacement achieved was $4 \mathrm{~mm}$, which is higher than reported by [1, 11, 14, 18]. A transistorized driver was designed to drive a solenoid by providing logical input at the base terminal of a transistor as shown in Figure 1 a. As soon as a logical input was available from the control unit to the base terminal of a transistor; solenoid actuated. Table 2 shows that $4 \mathrm{~mm}$ 


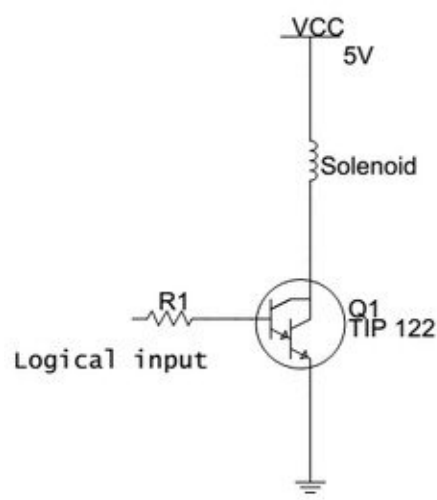

(a) Solenoid actuator driver circuit.

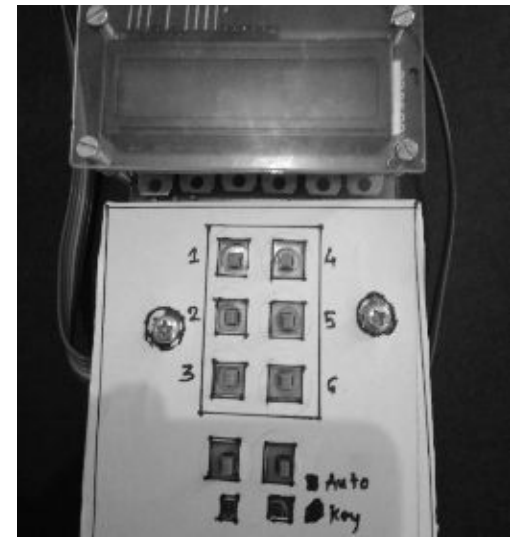

(b) Control unit front panel.

Figure 1: showing driver circuit and control unit.

displacement is achieved by an existing actuator in $11.8 \mathrm{~s}$ with an applied direct current of $0.5 \mathrm{~A}$. While the proposed actuator only takes $0.12 \mathrm{~A}$ and $10 \mathrm{~s}$ for similar displacement.

Table 2: Comparison of existing and proposed actuator performance parameter value

\begin{tabular}{|c|c|c|c|c|}
\hline & \multicolumn{2}{|c|}{$\begin{array}{c}\text { Existing } \\
\text { actuator }\end{array}$} & \multicolumn{2}{c|}{$\begin{array}{c}\text { Proposed } \\
\text { actuator }\end{array}$} \\
\hline $\begin{array}{c}\text { Displacment } \\
(\mathrm{mm})\end{array}$ & $\begin{array}{c}\mathrm{I} \\
(\mathrm{mA})\end{array}$ & $\begin{array}{c}\text { Time } \\
(\mathrm{s})\end{array}$ & $\begin{array}{c}\text { I } \\
(\mathrm{mA})\end{array}$ & $\begin{array}{c}\text { Time } \\
(\mathrm{s})\end{array}$ \\
\hline 0 & 100 & 2.3 & 0 & 0 \\
\hline 1 & 200 & 3.7 & 25 & 4 \\
\hline 1.5 & 250 & 4.3 & 45 & 5 \\
\hline 2 & 300 & 7.3 & 55 & 6.3 \\
\hline 2.5 & 350 & 8.2 & 75 & 7.4 \\
\hline 3 & 400 & 10.2 & 90 & 8.5 \\
\hline 3.5 & 450 & 11 & 105 & 9.6 \\
\hline 4 & 500 & 11.8 & 120 & 10 \\
\hline
\end{tabular}

\subsection{Performance test of an actuator array}

A $3 \times 2$ matrix was created using six solenoids close to each other in such a way that they will not affect the operation of neighbor pins. Two acrylic sheets of 3 $\mathrm{mm}$ thickness were used to host 6 solenoids as shown in Figure 2p. Each actuator is operated on $5 \mathrm{Vdc}$ and 0.12 A current. According to the excitation signal, maximum displacement achieved is $4 \mathrm{~mm}$ as shown in Figure $2 k$. Figure $2 \mathrm{a}$. shows a solenoid an unexcited state. Figure 2k shows a side view of the closely placed actuator. Figure $2 \mathrm{~d}$ is the top view of the tactile display showing the pin placing. Each pin was tested for its operation and combined six pins as well. This ensured the equal height of all pins when raised to avoid any misunderstanding because of adjacent pins. Also, the refresh rate is defined as actuation and de-actuation of pins; which is observed approximately $0.5 \mathrm{~s}$. However, during experimentation height (displacement) of the pin $2 \mathrm{~mm}$ was sufficient to interpret raised or un-raised pin from the reference surface. This reduced further power consumption of Braille cell to 1.6 W. It was one of the achievements of the proposed display.

The newly designed actuator has resulted in a re- duced current requirement to actuate to have $4 \mathrm{~mm}$ of displacement. It has also reduced the power consumption by each actuator. There is $75 \%$ reduction in the current required for actuation, which was achieved by increasing coil resistance by 4 times and $90 \%$ reduction in total power consumption by the tactile display as mentioned in Table 3

Table 3: Comparison between old and newly designed actuator parameters.

\begin{tabular}{|c|c|c|}
\hline Parameter & $\begin{array}{c}\text { Existing } \\
\text { actuator }\end{array}$ & $\begin{array}{c}\text { Proposed } \\
\text { actuator }\end{array}$ \\
\hline $\begin{array}{c}\text { Max current } \\
\text { requirement(A) }\end{array}$ & 0.5 & 0.120 \\
\hline $\begin{array}{c}\text { Operating DC } \\
\text { voltage(V) }\end{array}$ & 5 & 5 \\
\hline $\begin{array}{c}\text { Max displacement } \\
\text { (mm) }\end{array}$ & 4 & 4 \\
\hline $\begin{array}{c}\text { Length of } \\
\text { the rod(mm) }\end{array}$ & 3.2 & 4.2 \\
\hline $\begin{array}{c}\text { Coil resistance } \\
\text { (ohm) }\end{array}$ & 10 & 42 \\
\hline $\begin{array}{c}\text { Power consumption } \\
\text { by single actuator(W) }\end{array}$ & 2.5 & 0.6 \\
\hline $\begin{array}{c}\text { Power consumption } \\
\text { by six actuator(W) }\end{array}$ & 15 & 3.6 \\
\hline
\end{tabular}

\subsection{Control system design}

The developed circuit board uses an Arduino Mega board as a control unit to sequentially control solenoid actuator to represent the desired alphabet as shown in Figure 3 . The microcontroller was able to generate bilingual, that is English and Hindi alphabets and numbers. However, presently the evaluation is done for English alphabets only. The control unit has 6 keys used to write Braille letters on a tactile display as shown in Figure $1 \mathrm{~b}$. Two keys are provided to select the mode of operation, one for auto training or reading mode and the other for manual mode or writing mode. This module is accompanied by an LCD display for a normal trainer to check the key combinations pressed and the tactile pattern expected on the tac- 


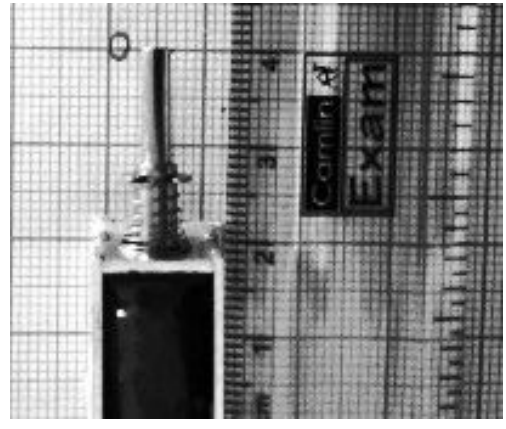

(a)

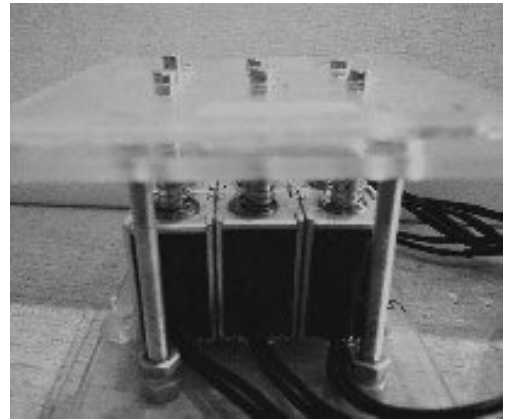

(c)

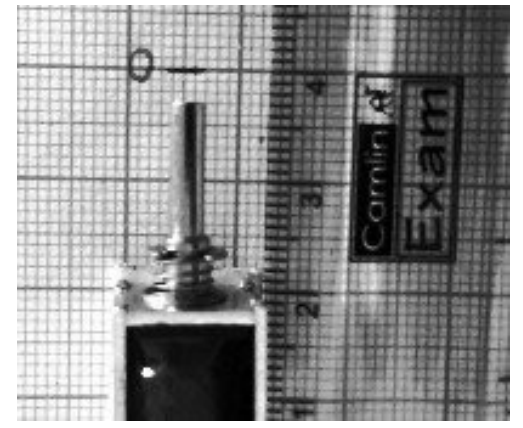

(b)

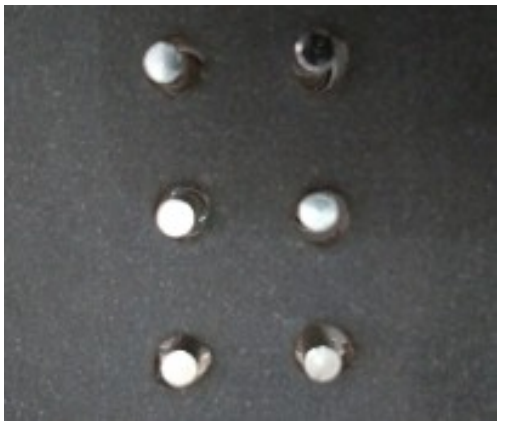

(d)

Figure 2: a) Actuator initial position, b) Actuator showing $4 \mathrm{~mm}$ displacement, c) Side view of developed six actuator cell showing compactness, d) Top view of six pin array to show pin to pin distance

tile display accordingly. A $3 \times 2$ matrix was created using six solenoids close to each other in such a way that they will not affect the operation of neighbor pins. The intention to use this arrangement is that even in the absence of an instructor; students can operate it in auto mode or learn Braille writing using keypress mode. The research work aims to test Braille reading by touching to pins of actuator and writing by key pressing electronically.

\section{Evaluation of tactile display}

A psychological experiment was carried out to understand the limitations of perception, accuracy, etc. The developed display was evaluated in the school Blind school for girls, Pune. Total 50 subjects, 25 blinds and 25 normal were selected for both experiments [26, 30]. Currently, restricted alphabets are successfully read and written by subjects. The delay of $1 \mathrm{~s}$ is set after every alphabet. This delay time was calculated through experimentation with the subjects during trials. The following two experiments were conducted.

- Tactile perception (Reading)

- Key Pressing sense (Writing)

\subsection{Experiment for tactile perception (reading)}

In Braille letters, the representations of letters such as e and i, d, h and j, s and t only differ by one dot. Thus, there is a possibility of misperception into the account with the developed display; alphabets were selected taking this in to account. The six dots formed by each actuator make up a display and raised or un-raised dots are felt by subjects by touching each pin. The raised height of each dot and the spacing between the dots play significant roles in the discrimination [30]. Intentionally selected 10 English alphabets were represented for subjects to touch and feel. Each subject was given the training to use the developed display for 5 minutes. They were instructed to use the index finger for touch and feel. Participating subjects were asked to read as rapidly as they could. Few samples manually highlighted for clarity as shown in Figure 3 English alphabets a, l, s, v, e, t, z, b, g, y were randomly presented. The time required to follow the alphabets with touch and reproduce them mentally and vocally is called reading time. For this experiment; time taken by each subject to read all ten alphabets by touch was considered as reading time. The time required to identify a letter as known before and realize the validity is called recognition time. For this experiment; time taken by all subjects to identify one alphabet was considered as recognition time.

The experiment aimed to measure the reading time and recognition time of the user. Reading time varies with user experience in reading Braille or the frequency of use of the tactile display. Minimum time for reading was observed to be $12 \mathrm{~s}$ by blind subjects and $11 \mathrm{~s}$ by normal subjects, while the maximum time was noted to be $22 \mathrm{~s}$ by blind and $20 \mathrm{~s}$ the normal as shown in Table 4 In an alphabet reading experiment, the time required to identify an alphabet is measured in seconds irrespective of the accuracy. Expected average 


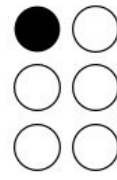

(a)

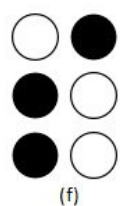

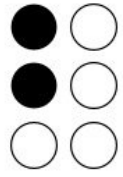

(b)

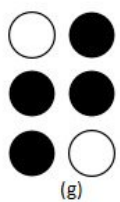

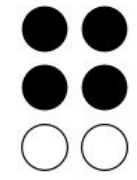

(d)

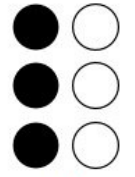

(e)

Figure 3: Manually highlighted (for clarity) alphabets presented on the developed display (a) a, (b) b, (c) e, (d)g, (e) l, (f) s, (g) t, (h) v, (i) y, (j)z

time to read 10 alphabets was assumed to be $10 \mathrm{~s}$. In the identification table; alphabets identified correctly were marked with 1 and incorrectly identified with 0 . The matrix was maintained as 10 alphabets in rows and 25 subjects in columns. During the recognition experiment; the average time expected was $1.5 \mathrm{~s}$ which was more than that of reading time. The column wise sum indicated reading time for all 10 alphabets of each subject and row wise sum indicated recognition time of each alphabet for all 25 subjects. Each subject had one attempt for each experiment.

Table 4: Reading and recognition time for blind and normal subjects.

\begin{tabular}{|c|c|c|c|c|}
\hline & \multicolumn{2}{|c|}{ Reading time (s) } & \multicolumn{2}{c|}{ Recognition time(s) } \\
\hline Subjects & Min & Max & Min & Max \\
\hline Blind & 12 & 22 & 28 & 54 \\
\hline Normal & 11 & 20 & 28 & 57 \\
\hline
\end{tabular}

As shown in Figure 4 English alphabet a, b, g were identified by all the subjects from each type. Alphabet $t$ has shown a less amount of correct identification compared to other letters. Figure 4 a shows the accuracy of alphabet identification in percentage. It shows that the letter a, b, g has shown $100 \%$ accuracy in identification by 50 subjects; while the letter $\mathrm{t}$ was only identified $80 \%$ accuracy. As shown in Figure 4 r the average reading time of 50 subjects was found to be 15.7 $\mathrm{s}$ using the developed tactile display. It is also required to test both types of subjects on the identification of each letter. Letter $t$ was identified by 19 blind persons and 21 normal persons out of 25 each. The reason for this was that blind persons missed dot number four.

Recognition time may vary with language.Figure $4 \mathrm{~d}$ shows recognition time for blind and normal subjects. The average recognition time required by 50 subjects was observed to be $39 \mathrm{~s}$. The total test time for the blind subject was $798 \mathrm{~s}$ for blind subjects and $764 \mathrm{~s}$ for normal subjects. During this experiment, it was observed that the minimum letter identification accuracy achieved was $80 \%$ and the maximum was $100 \%$. Blind subject no. 19 recorded the minimum time to all alphabets (12 s) while subject no. 2 recorded the highest time of $22 \mathrm{~s}$ for the same. Normal subject no. 25 recorded the minimum time to read all alphabets (11 s), while subject no. 2 recorded the highest time of $20 \mathrm{~s}$ for the same. As shown in Figure $4 \mathrm{~d}$ the recognition time for each alphabet by both types subjects was very small. The reason for this was observed to be the distance between adjacent pins.

\subsection{Experiment to test key pressing sense (writing)}

Key pressing sense testing is one of the major requirements to replace the existing Braille writing system. $3 \times 2$ keys were arranged in such a way that the key position representing Braille dots as usually they work with nails to form a dot. All of the participants involved have shown quite an interest as they were trying to relate this to their Braille writing technique. They pointed out that; their usual way of writing Braille letters is similar to the experimental process introduced here. However, reading is by reversing the page, which while reverses dot positions. the developed unit serves this aspect. When a particular key or combination of keys is pressed the respective Braille letter will be formed on the tactile display. The subjects are trained to press keys in a particular order to form the same Braille alphabet on a tactile display. The subjects had to touch the display and identify so that the representation is successful. This experiment was carried out immediately after Braille reading. Each subject used a test unit for 5 minutes to understand the keys. They were instructed to generate the same letters that they read in the previous experiment. The distance between the adjacent keys was $150 \mathrm{~mm}$ which is greater than the minimum discrimination distance required to sense pressed or un-pressed keys [30].

On the other hand, an invalid key combination in the set of alphabets is not displayed in a tactile form and a warning is generated on the LCD display for a normal trainer with a beep sound. Figure $4 \mathrm{~b}$ shows the response of subjects to the key pressing experiments. Total of 500 responses was recorded with 50 subjects and 10 alphabets. Assuming that each subject would 


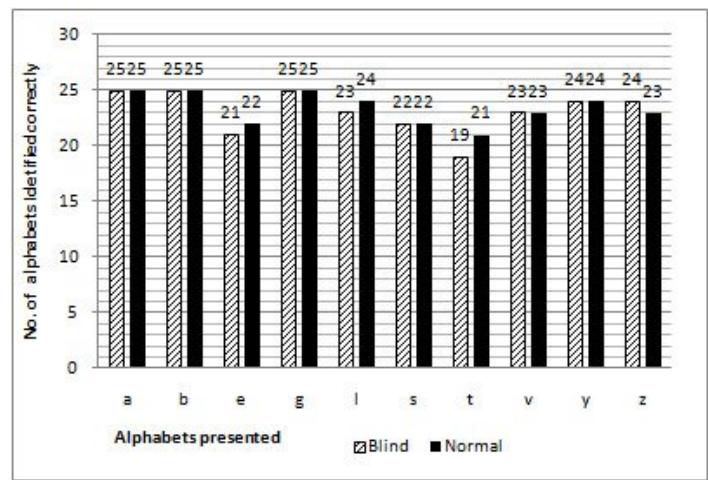

(a)

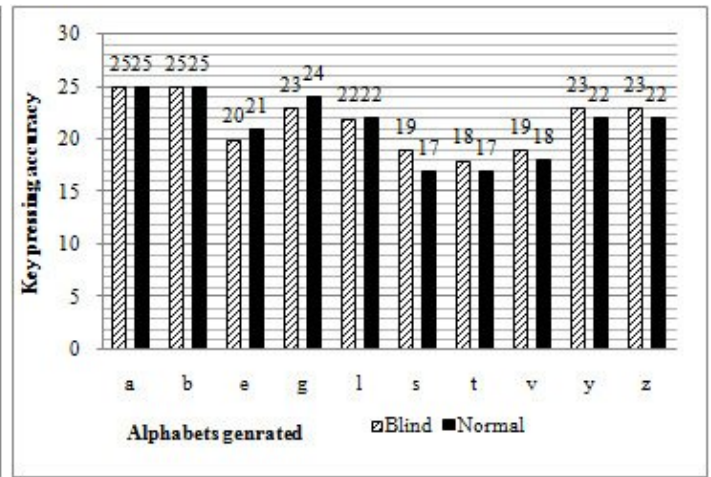

(b)

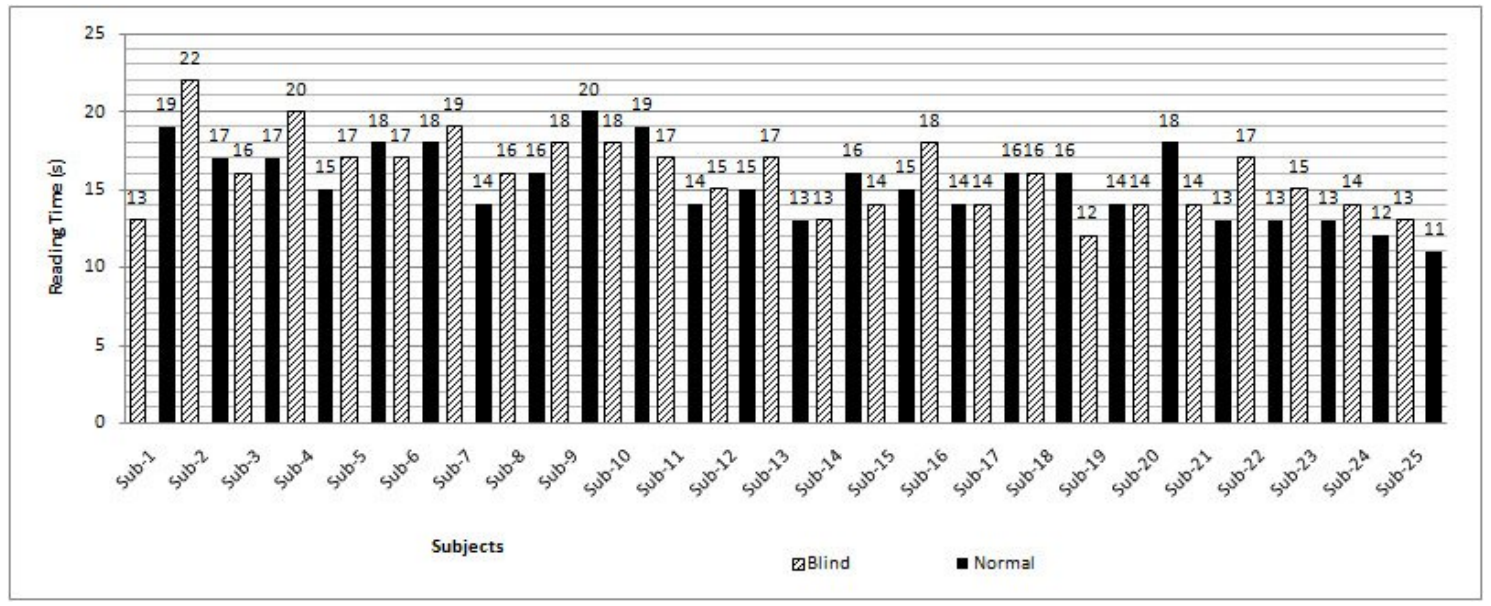

(c)

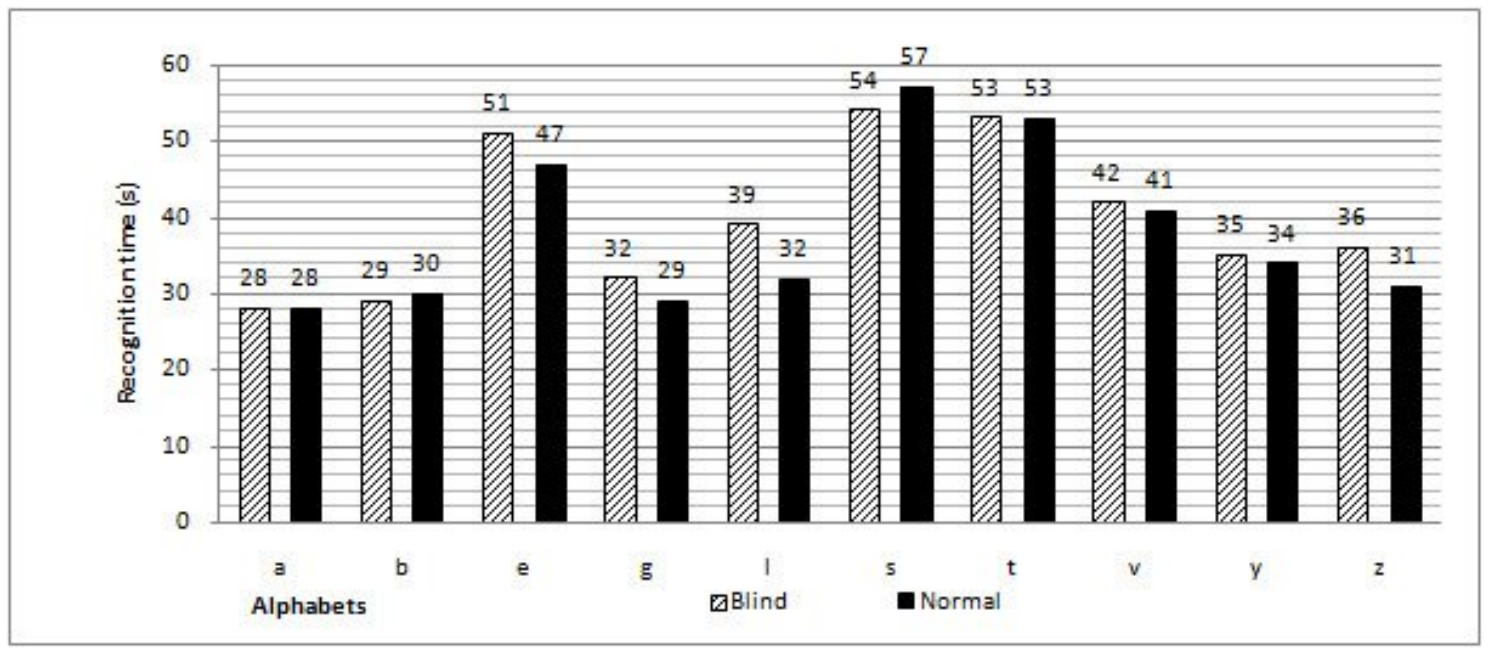

(d)

Figure 4: a) Number of alphabets identified correctly by each subject, b)Key pressing accuracy by both type of subjects,c) Reading time of each subject, d) Recognition time of each alphabet 
take approximately $1.5 \mathrm{~s}$ to form an alphabet on the display. The time estimated to conduct the test was 900 s. Time taken for completion of the entire test was 750 s. It was seen that on an average $86 \%$ of subjects pressed the correct keys to represent the target alphabet. In case of the letters $a$ and $b$, both types of subjects showed $100 \%$ success. In case of the letter s blind participants showed $76 \%$ success, while normal showed $68 \%$. The reason may be because of dot number five being mispressed by several subjects. In the case of the letter $\mathrm{g}$, normal subjects showed $96 \%$ accuracy while the blind showed only $92 \%$. The overall impression from this experiment was that the subjects a good sense of key pressing and that in the absence of trainer; they can use the developed system to learn alphabets through touch and feel. This experiment was validated through a tool known as a confusion matrix. Item analysis was also conducted to understand the degree of ease of perception using the developed model.

\section{Result and discussion}

In the identification test, a correctly identified letter was marked as 1 and others as 0 . The values obtained through identification for blind subjects and normal subjects have a very small difference, indicating that participants have a similar experienced in terms of touch and feel. An average of 23 subjects from each group identified alphabets with $93 \%$ accuracy. Alphabets $a, b, g$ were correctly identified by all of the participants, while alphabet $t$ was identified by the least number of participants. An average of 9 alphabets are identified by 13 blind and 14 normal subjects. The average time taken to read 10 alphabets was $16 \mathrm{~s}$ for blind subjects and $15 \mathrm{~s}$ for normal subjects. The reading rate was found to be 10 char/16 s. Overall reading accuracy was $93.9 \%$ for blind subjects and $94.74 \%$ for normal subjects it is listed in Table 5 . The reading rate was recorded as 1 char $/ 1.6 \mathrm{~s}$ for blind subjects and 1 char $/ 1.5 \mathrm{~s}$ for normal subjects. Although the reading rate was higher than the target rate, the reason for that was found to be the excessive closeness of tactile pins. The error rates recorded were $6.1 \%$ for blind subjects and $5.26 \%$ for normal subjects.

The recognition time taken was $798 \mathrm{~s}$ for all of the blind subjects combined and $764 \mathrm{~s}$ for all of the normal subjects combined. The average time taken to recognize 10 alphabets using developed tactile display was $40 \mathrm{~s}$ for 25 blind subjects $38 \mathrm{~s}$ for 25 normal subjects. For both types of subjects, the minimum recorded time to recognize 1 alphabet was $28 \mathrm{~s}$ and the minimum 10 alphabets with the developed display was $12 \mathrm{~s}$. The overall impression of the experiment was that there is very little difference in the recognition rate and reading rate. It indicates that some subjects may learn at a slower pace than the others and that some alphabets have fast perception while others require more time. In the experiment, an average reading or recognition time for 10 alphabets or 25 subjects was noted to be $1.6 \mathrm{~s}$.
Some subjects faced trouble in forming letters $t$ and $s$ by pressing keys due to the difference of one dot in the representations. They were unable to differentiate between keys and hence, pressed them an incorrect manner. Interaction with the subjects revealed that key pressing and then immediately reading may have led to some amount of confusion. There were two letters and b which were most correctly represented because they are consecutive and they involves less number of key pressing combinations. It was also observed that the distance between the keys during pressing and then the distance between the tactile pins significant affect accuracy. On an average, 8 alphabets were correctly presented by both types of participants. The accuracy calculated for key pressing sense was $86.6 \%$ for blind subjects and $85.2 \%$ normal subjects. The accuracy target was set to $90 \%$. A major observation was that the distance between push buttons (keys)reduced accuracy. Subjects were allowed only one attempt to form the desired alphabet. Recognition accuracy rates reported by previous developers for tactile displays varies from $60 \%$ to $90 \% ; 60 \%$ by [9], $80.83 \%$ by [5], $85 \%$ by [28], and $90 \%$ by [7]. The proposed work has achieved a rate of $95.7 \%$. To confirm achieved results two statistical techniques were used to analyze the data collected from these experiments.

- Confusion matrix technique

- Item analysis

\subsection{Confusion matrix}

Confusion matrix technique was applied to the collected data. Table 6 shows the presented patterns in rows and the answers given by subjects in the columns. Diagonal values are the responses of the subjects. The matrix shows that $a, b, g$ was presented to 50 subjects and all of them recognized them correctly. Alphabet $\mathrm{e}$ was presented to 50 participants out of which 43 identified it as 1 and 7 unexpectedly identified a letter; that was not listed in the test set. Alphabet 1 was presented to 50 participants and 3 out of them identified it as v. Similarly, alphabet $\mathrm{s}$ was presented to all participants and 6 out of them identified it as $t$. Alphabet $t$ was presented to all participants and 10 out of them identified it as $\mathrm{s}$. Alphabet $\mathrm{v}$ was presented to all participants; 4 out of them identified it as 1 . Alphabet $y$ is presented to all participants; 2 out of them identified it as $\mathrm{z}$. Alphabet $\mathrm{z}$ is presented to all participants 3 out of them identified it as $\mathrm{y}$.

The total 500 response were recorded as shown in Table 6. It shows the accuracy of $93 \%$. Misprediction is measured. The calculations show that mis-prediction was $7 \%$. This may be because the distance between pins is not as per suggestions were given by psychological experiments in [30]. The accuracy was high even though it was not par with Braille standards. This was because most of the subjects had been reading and writing Braille for more than 5 years [7]. The time required needs to be reduced further aligning the design with international Braille standards. 
Table 5: Comparison of performance parameters for blind and normal subjects.

\begin{tabular}{|c|c|c|}
\hline Parameter & Blind & normal \\
\hline $\begin{array}{c}\text { Identification } \\
\text { accuracy }(\%)\end{array}$ & 94 & 95 \\
\hline $\begin{array}{c}\text { Reading rate } \\
\text { (char per second) }\end{array}$ & $1 / 1.6$ & $1 / 1.5$ \\
\hline $\begin{array}{c}\text { Average reading } \\
\text { Time(s) }\end{array}$ & 16 & 15 \\
\hline $\begin{array}{c}\text { Average recognition } \\
\text { Time(s) }\end{array}$ & 40 & 38 \\
\hline $\begin{array}{c}\text { Key Pressing } \\
\text { Accuracy }(\%)\end{array}$ & 86.6 & 85.2 \\
\hline
\end{tabular}

Table 6: Confusion matrix: letters presented in rows and prediction in columns.

\begin{tabular}{|c|c|c|c|c|c|c|c|c|c|c|c|c|}
\hline \multicolumn{12}{|c|}{ Answers received } & \multirow[b]{2}{*}{ Total } \\
\hline \multirow{11}{*}{$\begin{array}{l}\vec{D} \\
\stackrel{D}{0} \\
0 \\
D \\
D \\
0\end{array}$} & & $\mathrm{a}$ & $\mathrm{b}$ & $\mathrm{e}$ & $\mathrm{g}$ & 1 & $\mathrm{~s}$ & $\mathrm{t}$ & $\mathrm{v}$ & $\mathrm{y}$ & $\mathrm{z}$ & \\
\hline & a & 50 & $0^{\mathrm{a}}$ & 0 & 0 & 0 & 0 & 0 & 0 & 0 & 0 & 50 \\
\hline & $\mathrm{b}$ & 0 & 50 & 0 & 0 & 0 & 0 & 0 & 0 & 0 & 0 & 50 \\
\hline & $\mathrm{e}$ & 0 & 0 & 43 & 0 & 0 & 0 & 0 & 0 & 0 & 0 & 43 \\
\hline & $\mathrm{g}$ & 0 & 0 & 0 & 50 & 0 & 0 & 0 & 0 & 0 & 0 & 50 \\
\hline & 1 & 0 & 0 & 0 & 0 & 47 & 0 & 0 & 3 & 0 & 0 & 50 \\
\hline & $\mathrm{s}$ & 0 & 0 & 0 & 0 & 0 & 44 & 6 & 0 & 0 & 0 & 50 \\
\hline & $\mathrm{t}$ & 0 & 0 & 0 & 0 & 0 & 10 & 40 & 0 & 0 & 0 & 50 \\
\hline & $\mathrm{v}$ & 0 & 0 & 0 & 0 & 4 & 0 & 0 & 46 & 0 & 0 & 50 \\
\hline & $\mathrm{y}$ & 0 & 0 & 0 & 0 & 0 & 0 & 0 & 0 & 48 & 2 & 50 \\
\hline & $\mathrm{z}$ & 0 & 0 & 0 & 0 & 0 & 0 & 0 & 0 & 3 & 47 & 50 \\
\hline & Total & 50 & 50 & 43 & 50 & 51 & 54 & 46 & 49 & 51 & 49 & 493 \\
\hline
\end{tabular}

\subsection{Facilitation and descrimination analy- sis}

Item analysis is a statistical method of judging the quality of an individual's discrimination power. It also yields the relationship of each item with the other items. Kelly T. L. proposed the technique to evaluate the facilitation value and discrimination index for a given data set [31]. The facilitation value $\left(F_{V}\right)$ is a measure of ease of perception? In this experiment, it indicates how easy it is to rightly identify a presented letter on a tactile display. A discrimination index $\left(D_{I}\right)$ indicates how well differentiating among different values? In our case, it is a measure of how well it discriminates amongst letters and dot positions.

$$
\begin{gathered}
F_{V}=\frac{\left(R_{U}+R_{L}\right)}{(2 \times n \times \text { max marks })} \\
D_{I}=\frac{\left(R_{U}-R_{L}\right)}{(n \times \text { max marks })}
\end{gathered}
$$

The given data set was arranged in descending order of marks obtained in the perception test. To calculate $F_{V}$ and $D_{I}$ the number of right and wrong answers are taken into account. According to [31] 27\% of the subjects are selected for the upper right $\left(R_{U}\right)$ answers segment and lower right $\left(R_{L}\right)$ answers segment of the group of subjects. There were 50 participants in total. Hence, 14 subjects were each from the top and the bottom of the list in descending order of total marks respectively called $R_{U}$ and $R_{L}$. In the test, each correct answer gave one mark, with 10 letters presented, the maximum possible score was 10 . The sum of $R_{U}$ marks is 140 and the sum of $R_{L}$ marks 114 as reported in Table 7

Table 7: Mark obtained during identification test ordered from high to low.

\begin{tabular}{|c|c|c|c|}
\hline \multicolumn{2}{|c|}{ Upper right answer } & \multicolumn{2}{c|}{ Lower right answer } \\
\hline Subject no. & Marks & Subject no. & Marks \\
\hline 5 & 10 & 33 & 9 \\
\hline 7 & 10 & 34 & 9 \\
\hline 8 & 10 & 36 & 9 \\
\hline 11 & 10 & 38 & 9 \\
\hline 13 & 10 & 43 & 9 \\
\hline 17 & 10 & 48 & 9 \\
\hline 18 & 10 & 2 & 8 \\
\hline 19 & 10 & 6 & 8 \\
\hline 20 & 10 & 14 & 8 \\
\hline 21 & 10 & 44 & 8 \\
\hline 22 & 10 & 46 & 8 \\
\hline 23 & 10 & 10 & 7 \\
\hline 24 & 10 & 12 & 7 \\
\hline 26 & 10 & 27 & 6 \\
\hline Total & 140 & Total & 114 \\
\hline
\end{tabular}

Facilitation value is calculated as per Eq,1, and discrimination index is calculated as per Eq 2 , where $n$ indicates the number of participants. 
Table 8: Comparison with previously developed systems (NR:represents not reported).

\begin{tabular}{|l|l|l|l|l|l|}
\hline Parameter (unit) & {$[5]$} & {$[29]$} & {$[28]$} & {$[18]$} & $\begin{array}{l}\text { proposed } \\
\text { work }\end{array}$ \\
\hline Display Size(mm) & $12 \times 12 \times 12$ & $6.4 \times 8.7$ & $15 \times 15 \times 15$ & $40 \times 40$ & $15 \times 30 \times 30$ \\
\hline Cell size & $3 \times 3$ & $8 \times 1$ & $3 \times 2$ & NR $^{\mathrm{a}}$ & $3 \times 2$ \\
\hline Stroke or displacement $(\mathrm{mm})$ & 1 & 1 & NR & NR & 4 \\
\hline Type of actuator & Piezo & Piezo & SMA wire & Solenoid & Solenoid \\
\hline Force (N) & 0.2 & NR & NR & NR & 1 \\
\hline Operating Frequency (Hz) & $0-5$ & 200 & 100 & NR & 0.2 to 5 \\
\hline Weight(gm) & 2.5 & NR & NR & NR & 150 \\
Reading Accuracy (\%) & 80.83 & 90.0 & 85 & NR & 94 \\
\hline Duration of trial (s) & 5.5 & $300-600$ & 10 & NR & 750 \\
\hline Presented alphabet $\backslash$ numbers & 0 to 9 & NR & 10 & NR & 10 \\
& Numbers & & Alphabets & & Alphabets \\
\hline Power per cell(W) & NR & NR & 0.08 & 2 & 1.6 \\
\hline
\end{tabular}

$F_{V}=0.94$ (Ideal value $=1$ and desired value is between 0.2 and $0.85[31]$ ) and $D_{I}=0.11$ (Ideal value $=0$ and desired value is between 0.3 and 0.65 ). Thus, $F_{V}$ quite high and discrimination index is well within desired range.

\section{Summary}

A detailed comparative for parametric analysis is carried out for the proposed work in this paper and displays implemented by [5], [18], [28], [29] in terms of dimensions, mechanical considerations, and performance parameters and reported in Table 8 It shows that displacement is highest in comparison with literature and power consumption is least. These displays were designed in a similar line. It was found that the proposed display is efficient in presenting alphabets, and has shown the best discrimination in identifying characters within a small duration. The system is capable of facilitating the need for intended users. The duration of the trial was long because it was the first tactile experience for the users. It was learnt that training is required for the first use. To overcome this issue the proposed prototype was evaluated and experimented to assess every minute parameter.

\section{Conclusion}

In this paper, a prototype module for the novel $3 \times 2$ tactile display using 6 solenoid actuators was designed, implemented and evaluated. The extensive psychological experiments carried out show that the accuracy is $93 \%$ for the selected random alphabets. The reading time is 10 char per 16 seconds, and the recognition time is $31 \mathrm{~s}$. The average reading time is about 15.7 $\mathrm{s}$ the average recognition time achieved is $39.3 \mathrm{sec}-$ onds. Facilitation value of the developed display is $94 \%$, which is close to the desired value. The discrimination index is low, suggesting that the developed display has good discrimination amongst various characters identification tactile systems. The cost of the developed system is approximately $\$ 22$. Obtained results confirmed the superior performance of proposed display as compared to existing displays.

Conflict of Interest The authors declare no conflict of interest.

Acknowledgement(s) This work was supported by the Board of Colleges and University Development (BCUD, Savitribai Phule Pune University (SPPU), Pune, India (Proposal number 15ENG001916). Special thanks to Management and principal of The Poona School and home for blind girls, Kothrude, Pune, and Maharashtra for conducting the test in the school.

\section{References}

[1] Taylor PM., Moser A., Creed A., "A sixty-four element tactile display using shape memory alloy wires" Elsevier Displays., 18(3,163-168, 1998.doi.org/10.1016/S0141-9382(98)00017-1

[2] Pruittikorn smithmaitrie, Jinda Kanjantoe, Pichaya Tandayya, "Touching force response of piezoelectric Braille cell" Disability and Rehabilitation: Assistive technology, 6,360365,2008. doi.org/10.1080/17483100802281442

[3] Velazquez Ramiro, Hermes Hernandez, Enrique Preza, "A portable eBook reader for the blind" 32nd Annual International Conference of IEEE Engineering in Medicine and Biology Society (EMBS), Buenos Aires, Argentina, Sep 2010. DOI: 10.1109/IEMBS.2010.5626218

[4] Ang Beng Seng, Zuraini Dahari, Othman Sidek, "Design and analysis of thermal microactuator". European Journal of Scientific Research., 35(2), 281-292, 2009.

[5] Kyung Ki-Uk, Jun-Young Lee, Junseok Park "Design and applications of a pen-like haptic interface with texture and vibrotactile display" Frontiers in the Convergence of Bioscience and Information Technologies, FBIT 2007(IEEE), Jeju Island, Korea, 2007. DOI: 10.1109/FBIT.2007.92

[6] Wu X., Zhu H., Kim S H. Mark A., "A portable pneumaticallyactuated refreshable braille cell". IEEE Conference on SolidState Sensors, Actuators, and Microsystems (TRANSDUCERS 2007), Lyon, France, Jun 2007. DOI: 10.1109/SENSOR.2007.4300407 
[7] Kyung Ki-Uk, Minseung Ahn, Dong-soo Kwon,Shrinivasan M. A., "A compact planar distributed tactile display and effects of frequency on texture judgment" Advanced Robotics,20(5),563-580, 2006. doi.org/10.1163/156855306776985540

[8] Takamiya Makoto, Tsuyoshi Sekitani, Yusaku Kato, Hiroshi Kawaguchi, Takao Someya, Takayasu Sakurai. "Low power and flexible braille sheet display with organic FET's and plastic actuators", International Conference On IC Design and Technology (ICICDT'06) IEEE, Padova, Italy, May2006. DOI: 10.1109/ICICDT.2006.220831

[9] Choi $\mathrm{H}$ R, Lee $\mathrm{S}$ W, lung $\mathrm{K} \quad \mathrm{M}$, Koo I C,Lee S I,Choi H G,Jeon J W,Nam J D, "Tactile display as a braille display for the visually disabled" Proceedings IEEE/RSJ International conference on Intelligent Robots and Systems (IROS 2004),Sendal, Japan,nSep 2004. DOI: 10.1109/IROS.2004.1389689

[10] Matsunaga T, Totsu K, Esashi M, Haga Y., “2-D and 3-D tactile pin display using SMA micro-coil actuator and magnetic latch", The 13th International Conference on Solid-State Sensors, Actuators, and Microsystems, Digest of Technical Papers (TRANSDUCERS'05 IEEE),Seoul, South Korea, June 2005. DOI: 10.1109/SENSOR.2005.1496422

[11] Wagner Christopher R, Susan J Lederman, Robert D Howe, "A tactile shape display using RC servo motors", IEEE Proceedings of 10th Symposium on Haptic Interfaces for Virtual Environment and Teleoperator Systems,(HAPTICS, 2002), Orlando, FL, USA, March 2002. DOI: 10.1109/HAPTIC.2002.998981

[12] Konyo Masashi, Satoshi Tadokoro, Toshi Takamori, "Artificial tactile feel display using soft gel actuators", Proceedings of IEEE international conference on Robotics and $\mathrm{Au}$ tomation, (ICRA'00), San Francisco, CA, Apr 2000. DOI: 10.1109/ROBOT.2000.845250

[13] Smithmaitrie Pruittikorn, Jinda Kanantoe P., "Analysis and design of Piezoelectric Braille display", Rehabilitation Engineering, InTech, 2009. Available from: https://www.intechopen.com

[14] Velazquez R, Pissaloux E, Szewczyk,Hafez M., "Miniature shape memory alloy actuator for tactile binary information display", Proceedings of IEEE International Conference on Robotics and Automation,ICRA 2005, Barcelona, Spain, Apr 2005, DOI: 10.1109/ROBOT.2005.1570302.

[15] Mosley M, Constantinos Mavroidis, Charles Pfeiffer, "Design and dynamics of a shape memory alloy wire bundle actuator",Proceedings of the ANS, 8th Topical Meeting on Robotics and Remote Systems,Mar 1999. DOI:10.1.1.41.4629

[16] Lee Jun Su, Stepan Lucyszyn, "A micromachined refreshable Braille cell", Journal of Microelectromechanical Systems, 14(4),673-682, 2005. DOI: 10.1109/JMEMS.2005.845415

[17] Nobels Tiene, Frank Allemeersch, Kay Hameyer, "Design of a high power density electromagnetic actuator for a portable Braille display", Proceedings of 10th international power electronics \& motion control conference, EPE-PEMC, 2002.

[18] Sarah F Frisken Gibson, Paul Bach Y Rita, Willis J TOMPKINS,John G Webster, "A 64-solenoid, four-level fingertip search display for the blind", IEEE Transactions on Biomedical Engineering (BME), 34(12),963-965, 1987. DOI: 10.1109/TBME.1987.325937
[19] Deng Kai, Eniko T Enikov, P Marek, "A shape display method based on electromagnetic localization and actuation,PIERS Online,26(2),157-160, 2010.

[20] Francesca Sorgini, Renato Calio, Maria Chiara Carrozza,Calogero Maria Oddo, "Haptic-assitive technologies for audition and vision sensory disabilities",Haptic-assitive technologies for audition and vision sensory disabilities, Disability and rehabilitation: Assistive technology; 2017. DOI: 10.1080/17483107.2017.1385100.

[21] Dominic R Labbe, Guy Santerre, Sabastien McKenzie Faucher et a.1 . US patent no.9,965,974 B2.Drummonville(Quebec), CA, 2018.

[22] Shyam Shah.(2018).US patent no. US2018/0033336A1, Ahemadabad, India.

[23] Luzhnica G, Veas E., "Investigating Interactions for Text Recognition Using a Vibrotactile Wearable Display",In 23rd International Conference on Intelligent User Interfaces ACM,453-465,Tokyo, Japan, 2018. DOI:10.1145/3172944.3172951

[24] Leonardis D, Loconsole C.,"Braille cursor: An innovative and affordable refreshable braille display designed for inclusion", In 10th International Conference on Applied Human Factors and Ergonomics Springer, 302-311, Washington DC, USA, Jul 2019. DOI:10.1007/978-3-319-94622-129.

[25] Lu Zhiyun, "Fabricating One Cell of Refreshable Braille Display Using New Bistable Electroactive polymers. MS Theses University of california; 2017; Los Angeles.

[26] V B Vaijapurkar, Y Ravinder, "A survey on recent trends and technologies in tactile interfaces with sensing perspective",Proceedings of annual IEEE India Conference (INDICON 2015),New Delhi, India, Dec 2015. DOI: 10.1109/INDICON.2015.7443158

[27] Sara Demain, Cheryl D Metcalf, Geoff Merrett V, Deyi Zheng, Sarah Cunningham, "A narrative review on haptic devices: relating the physiology and psychophysical properties of the hand to devices for rehabilitation in central nervous system disorders", Disability and Rehabilitation: Assistive Technology, 8(3),181-189, 2013.Doi/abs/10.3109/17483107

[28] Feng Zhao, Changan Jiang, Hideyuki Sawada, "A novel Braille display using the vibration of SMA wires and the valuation of Braille presentations", Journal of Biomechanical Science and Engineering Japan,7(4),416-432, 2012. https://doi.org/10.1299/jbse.7.416

[29] Joseph Luk, Jerome Pasquero, Shanon little,MacLean Karon, Lvesque Vincent,Hayward Vincent, "A role for haptics in mobile interaction: initial design using a handheld tactile display prototype", Proceedings of CHI 2006 conference on Human Factors in computing systems ACM,22-27, Montreal, Canada,2006 Apr. DOI:1124772.1124800

[30] Kenneth O Johnson, John R Phillips, "Tactile spatial resolution-I, two-point discrimination, gap detection, grating resolution, and letter recognition",Journal of Neurophysiology, 46(6),1177-1192, 1981. DOI:abs/10.1152/jn.1981.46.6.1177

[31] Kelly T L., "The selection of upper and lower groups for the validation of test terms", Journal of Educational psychology, 46(6),1981. Doi=10.1037\%2Fh0057123 